\section{Neue Abteilung „,Technik- bedingte Stoffströme" am Institut für Technische Chemie des Forschungszentrums Karlsruhe}

von Liselotte Schebek, ITC

Das Institut für Technische Chemie (ITC), eines von 17 wissenschaftlichen Instituten des Forschungszentrums Karlsruhe (FZK), umfasst die drei experimentell und verfahrenstechnisch ausgerichteten Bereiche Chemisch-Physikalische Verfahren, Wasser- und Geotechnik und Thermische Abfallbehandlung. Im Herbst 1999 wurde eine neue Zentralabteilung gegründet, die bereichsübergreifend das Querschnittsthema „Technikbedingte Stoffströme“ bearbeitet und Recherche- und Bewertungsmethoden zur Untersuchung technischer Verfahren und ihres Umfelds bereitstellt. Die neue Abteilung wird projektorientierte Arbeiten durchführen, bei denen wissenschaftliche Erkenntnisse über technikbedingte Stoffströme unmittelbar in Kooperationen mit verfahrenstechnischen Entwicklungsarbeiten genutzt werden können.

Aufgabenstellung der neuen Zentralabteilung „Technikbedingte Stoffströme“ am ITC ist die wissenschaftliche Untersuchung relevanter Stoffströme der Industriegesellschaft und der mit ihnen verknüpften technischen Prozesse im Hinblick auf eine nachhaltige industrielle Entwicklung. Vor dem Hintergrund der Einbindung in ein chemisch-technisches Institut steht dabei der Beitrag von Technik und Naturwissenschaft zur nachhaltigen Entwicklung im Zentrum der Arbeiten. Der interdisziplinäre Charakter des Begriffes der Nachhaltigkeit, wie er in der umweltpolitischen Diskussion mit den drei Dimensionen Ökologie, Ökonomie und Soziales geprägt wurde, soll jedoch sowohl in der Themenauswahl als auch bei der Bewertung der Arbeitsergebnisse seinen Niederschlag finden. Insbesondere wird hierzu innerhalb des Forschungszentrums Karlsruhe eine intensive Zusammenarbeit mit dem Institut für Technikfolgenabschätzung und Systemanalyse (ITAS) aufgebaut.

Die Konzeption als bereichsübergreifende Zentralabteilung am ITC beinhaltet eine enge
Kooperation mit den experimentellen und verfahrenstechnischen Bereichen: Optimierungspotentiale im Hinblick auf die Nachhaltigkeit technischer Prozesse können idealer Weise im Rahmen der Verfahrensentwicklung implementiert werden; gleichzeitig müssen für die Wettbewerbsfähigkeit innovativer, ökologisch vorteilhafter Verfahren frühzeitig Informationen über das Umfeld zur Charakterisierung des angestrebten Marktes zur Verfügung gestellt werden. Über gemeinsame themenorientierte Projekte der Zentralabteilung mit Verfahrensentwicklern wird eine unmittelbare Nutzung der wissenschaftlichen Erkenntnisse über technikbedingte Stoffströme im Rahmen der F\&EArbeiten des Forschungszentrums ermöglicht. Insbesondere bei Stoffstromanalysen zu ausgewählten technischen Verfahren wird eine Kooperation mit dem ITAS erfolgen.

Die Arbeitsmethoden der Zentralabteilung umfassen die Recherche und Aufbereitung von Informationen sowie die Bewertung dieser Informationen. „Standortvorteil“ bezüglich der Recherche sind die am Forschungszentrum Karlsruhe bereitgestellten Zugriffsmöglichkeiten auf umfangreichen Datenbanken; essentiell ist darüber hinaus die Beschaffung von Primärinformationen über direkte Kontakte zu Akteuren der Wirtschaft, der Entsorgungsbranche und Verbänden sowie in- und ausländischen Forschungsinstituten, wie sie auch im Rahmen gemeinsamer Forschungsvorhaben aufgebaut werden sollen.

Bewertungsmethoden werden insbesondere aus dem Bereich der Ökobilanzierung zur Verfügung gestellt. Hier sollen zum einen bereits entwickelte Methoden - auch in Form entsprechender Software - genutzt werden, aber auch eine Anpassung oder Weiterentwicklung dieser Methoden auf die spezifischen Bedürfnisse bei der Anwendung im Bereich der Forschung und Entwicklung ist Thema der Forschungsarbeiten.

Die Leitung der Zentralabteilung Technikbedingte Stoffströme ist verbunden mit einer Professur für Industrielle Stoffkreisläufe am Institut für Wasserversorgung, Abwassertechnik, Abfalltechnik, Umwelt- und Raumplanung der Technischen Universität Darmstadt, Fachbereich Bauingenieurwesen, die das Thema industrieller Umweltschutz als Querschnittsaufgabe in Forschung und Lehre ver- 
treten wird. Angebunden an diese Professur wird weiterhin die wissenschaftliche Leitung einer geplanten Forschungsstelle Industrielle Stoffkreisläufe der Hessischen Industriemüll $\mathrm{GmbH}$, Wiesbaden, an der praxisorientierte Themen zu industriellen Abfallströmen behandelt werden sollen.

Auf Basis einer gemeinsamen Berufungskommission nach dem ,Jülicher Modell“ wurde Frau Dr. Liselotte Schebek mit der Abteilungsleitung sowie ab Sommersemester 2000 mit der Übernahme der Professur an der TU Darmstadt beauftragt. Frau Dr. Schebek studierte Chemie an der TU Darmstadt und promovierte als Mitarbeiterin der Abteilung Biogeochemie des Max-Planck-Instituts für Chemie in Mainz mit einer Untersuchung über Organometallverbindungen in aquatischen Systemen. Sie arbeitete als wissenschaftliche Mitarbeiterin des Öko-Instituts e.V. im Bereich Industrielle Abfallwirtschaft und war seit 1990 als Projektleiterin der Hauptabteilung Umweltschutz der Lahmeyer International GmbH (seit 1998 ERM Lahmeyer International $\mathrm{GmbH}$ ) tätig. Arbeitsschwerpunkte waren Sonderabfallwirtschaft, Vermeidung und Verwertung industrieller Abfälle, produktionsintegrierter Umweltschutz und Umweltmanagement; u.a. führte Frau Dr. Schebek das Projektmanagement für das mehrjährige Beratungsprogramm zur Reststoffvermeidung und -verwertung in Industrie und Gewerbe des Umweltministeriums Baden-Württemberg durch ${ }^{1}$ und bearbeitete in jüngster Zeit als Verbundprojekt ein Forschungsvorhaben zur EG-Öko-AuditVerordnung für das Hessische Umweltministerium $^{2}$.

\section{Anmerkungen}

1) Greiner, D., Lipfert, H. L. und Schebek, L., 1997: Verminderung von Abfällen aus badenwürttembergischen Industrie- und Gewerbebetrieben. In: Müll und Abfall, 9/1997, S. 520527.

2) Ankele, K., Fichter, K., Heuvels, K., Rehbinder, E. und Schebek, L., 1998: Fachwissenschaftliche Untersuchung der Wirksamkeit der EG-Öko-Audit-Verordnung. In: Umweltwirtschaftsforum, 4/1998, S. 38-44.

\section{Kontakt}

Dr. Liselotte Schebek

Institut für Technische Chemie

Zentralabteilung Technikbedingte Stoffströme

Forschungszentrum Karlsruhe

Postfach 3640, D-76021 Karlsruhe

Tel.: + 49 (0) 7247/82-6561

Fax: + 49 (0) 7247/82-4373

E-Mail: Liselotte.Schebek@itc-tab.fzk.de

\section{Gesetzesfolgenabschätzung am Forschungsinstitut für öffentliche Verwaltung}

\author{
von Götz Konzendorf, Forschungsinstitut \\ für öffentliche Verwaltung, Speyer
}

Im Zuge der Verwaltungsmodernisierung und der Staatsreform wird auch der Rechtsetzungsprozess optimiert. Gesetzesfolgenabschätzung (GFA), die sich in ein prospektives, ein begleitendes und ein retrospektives Modul unterteilen lässt, nimmt hierbei eine zentrale Rolle ein. Im folgenden Beitrag werden die Module der GFA sowie Ansätze zu ihrer Implementation in das politisch-administrative System dargestellt.

Das Thema Gesetzesfolgenabschätzung hat Konjunktur. Dies war nicht immer so. Zwar gab es schon zu Beginn der 80er Jahre einige Aktivitäten zur Optimierung des Rechtsetzungsprozesses durch Test- und Prüfverfahren von Gesetzentwürfen, doch erlosch das Interesse an diesen Instrumenten nach kurzer Zeit weitgehend. Zur gleichen Zeit wurden im Bund und in den Ländern Prüffragen für Rechtsvorschriften erlassen, ihre Anwendung kann aber nicht befriedigen: so ist es z.B. gängige Praxis, bei der Frage nach den entstehenden Kosten „keine“ anzugeben. Deshalb werden von Seiten der politischen Führung (z.B. Bundesinnenminister Otto Schily; Ministerpräsident Kurt Beck), von Teilen der öffentlichen Verwaltung, von Vertretern der Wirtschaft (z.B. BDI) und auch von Verwaltungswissenschaftlern Gesetzesfolgenabschätzungen gefordert. 